\title{
Comparison of ankle-brachial pressure index and pulse wave velocity as markers of cognitive function in a community-dwelling population
}

\author{
Norio Sugawara*1, Norio Yasui-Furukori2, Takashi Umeda33, Ayako Kaneda2, Yasushi Sato1, Ippei Takahashi³, \\ Masashi Matsuzaka ${ }^{3}$ Kazuma Danjo ${ }^{3}$, Shigeyuki Nakaji ${ }^{3}$ and Sunao Kaneko ${ }^{2}$
}

\begin{abstract}
Background: Vascular factors have been implicated in the development of cognitive decline and dementia. The purpose of this study is to determine the association of the Ankle Brachial pressure Index (ABI) and brachial-ankle Pulse Wave Velocity (ba-PWV) to cognitive impairment in a community-dwelling population.

Methods: The ABI and ba-PWV were measured using the volume-plethymographic apparatus in 388 subjects aged 60 years old and over. The Mini-Mental State Examination was also employed to measure global cognitive status. The effectiveness of the $A B I$ and ba-PWV as putative markers of cognitive impairment were determined by using a multiple logistic regression analysis after adjusting for confounding factors.

Results: Subjects with poor cognition were significantly older and less well educated than those with normal cognition. According to the multiple logistic regression analysis, the lowest ABI tertile was found to be a significant independent risk factor $(\mathrm{OR}=3.19,95 \% \mathrm{Cl}=1.30$ to 7.82$)$ of the cognitive impairment, whereas the highest brachialankle PWV tertile was not.

Conclusions: A low $\mathrm{ABI}$ was an independent risk factor for cognitive impairment in community-dwelling older populations, whereas a high ba-PWV may not be. Further research will be required to analyze ABI and PWV with greater accuracy.
\end{abstract}

\section{Background}

The cognitive impairment is an important health issue contributing to disability [1], morbidity [2] and mortality [3]. The identification of clinical markers predicting cognitive impairment in elderly is often considered useful in easing the public health burden of poor cognition, as aging is associated with an increased risk of cognitive impairment.

Vascular factors have been implicated in the development of cognitive decline [4] and dementia [5]. As common quantitative assessments of arterial health, the Ankle Brachial pressure Index (ABI) and Pulse Wave Velocity (PWV) are often considered to determine blocked arteries and arterial stiffness respectively, using non-invasive measures.

\footnotetext{
* Correspondence: nsuga3@yahoo.co.jp

1 Department of Psychiatry, Hirosaki-Aiseikai Hospital, Hirosaki, Japan Full list of author information is available at the end of the article
}

The ABI is the ratio of the ankle and the brachial systolic blood pressure and is used to assess the severity of arterial occlusion in the leg, and a reduction of ABI suggests the presence of peripheral arterial disease due to atherosclerosis. It is also known that atherosclerosis in the lower legs represent just one manifestation of a similar pathology in other arterial systems [6,7]. A number of previous studies $[4,8,9]$ on the relationship between ABI and cognitive function were carried out, in populationbased designs. Breteler et al [10] had observed that peripheral arterial disease was associated with lower average mental status scores and shifts in score distribution. In a large US community-based study [8], a low ABI was associated with the decline of cognitive function over 7 years of follow up. Other cohort studies including the Edinburgh Artery study and the Honolulu-Asia Aging study, showed that a lower ABI had predictive value for 
future risk of cognitive impairment [4] and increased the risk of dementia [9].

Another assessment for arterial stiffness is PWV, in which the velocity of the pulse wave is measured as it travels a given distance between 2 sites along the arterial system. Since Mizushima et al. [11] first reported that PWV was higher for individuals with vascular dementia than those without dementia; the PWV has been recognized as a risk factor of dementia [12,13]. According to a result obtained in a cross-sectional design study [14-16], a higher PWV has also been related to a decrease in performance on screening measures of cognitive function, such as the Mini-Mental State Examination (MMSE). The longitudinal effects of PWV to verbal learning, delayed recall, non verbal learning [17] and global cognitive function [18] were also reported.

These findings contribute to the growing body of evidence on correlation between arterial stiffness and cognitive impairment. Recently, a measurement device which can simultaneously measure the ABI and brachial-ankle PWV has become available [19]. However, the association between these 2 putative markers for the assessment of cognitive impairment in a community-dwelling population has not yet been determined.

In this study, the ABI and ba-PWV were compared as markers of cognitive impairment in a community-dwelling population, and MMSE was used to assess cognitive status. The ABI and PWV were measured as indicators of arterial structure and function. The present study is considered as the report to obtain a comparative data between the ABI and ba-PWV as markers of cognitive function in a community-dwelling population.

\section{Method}

\section{Participants}

The subjects were 388 volunteers (60 years old and over; 139 males and 249 females) who participated in the Iwaki Health Promotion Project in 2008. The data collection for this study was approved by the Ethics Committee of Hirosaki University School of Medicine and all subjects had provided written informed consent before participating in the project. The demographic data (age, sex, amount of education) and life style (smoking, drinking) were obtained from self-questionnaires and interviews. The height and weight of subjects were measured, and body mass index (BMI) was calculated. Low-density lipoprotein (LDL) cholesterol, triglyceride and HbAlc were also measured by standard analytical techniques.

\section{$A B I$ and brachial-ankle PWV measurements}

The ankle and brachial pressures were measured using the volume-plethymographic apparatus (form PWV/ABI, COLIN Co. Ltd., Tokyo, Japan). In addition to recording the limb lead ECG and mechano-cardiograms were simultaneously recorded by attaching blood pressure cuffs with a tonometic sensor to the upper arm and ankle.

The ABI was determined as the ratio of ankle systolic blood pressure to brachial systolic blood pressure. To calculate the $\mathrm{ABI}$, the brachial pressure was measured in the left arm, and the ankle pressure was measured for both the left and right sides with subjects in the supine position and the lowest value of the ABI was taken [9].

The brachial-ankle PWV (ba-PWV) was calculated by time-phase analysis. The time interval between the wave front of the brachial waveform and that of the ankle waveform was defined as the time interval between the brachium and ankle $(\Delta \mathrm{Tba})$. The distance between sampling points of ba-PWV was calculated automatically according to the height of the subject. The path length from the suprasternal notch to the brachium (Lb) was obtained from superficial measurements and was expressed using the following equation: $\mathrm{Lb}=0.2195 \times$ height of the patient (in $\mathrm{cm}$ ) -2.0734 . The path length from the suprasternal notch to the ankle (La) was obtained from superficial measurements and was expressed using the following equation: $\mathrm{La}=(0.8129 \times$ height of the patient (in $\mathrm{cm})+12.328)$. Finally, the following equation was used to obtain ba-PWV: ba-PWV = (LaLb)/ $\Delta$ Tba [20]. In this study, a higher value of ba-PWV was employed.

\section{Assessment of cognitive function}

The Mini-Mental State Examination (MMSE) was given to all participants to measure their global cognitive status. This test assesses orientation to place and time, short term memory, episodic long-term memory, subtraction, ability to construct a sentence, and oral language ability. The maximum score was set as 30 , and poor cognition was defined as a score of less than 24 [21]. Subjects were divided into 2 groups: control group (MMSE^$\left.{ }^{\wedge} 4\right)$ and the group with subjects having a cognitive impairment (MMSE\%23).

\section{Statistical Analysis}

Data are presented as mean \pm SD. A value of $\mathrm{p}<0.05$ was considered significant. Student's unpaired t test for continuous variables or chi-square test for categorical variables was used to evaluate the difference in variables between subjects with MMSE\%23 and those with $\mathrm{MMSE}^{\wedge} 24$. The subjects were divided into 3 groups according to their $\mathrm{ABI}$ and ba-PWV tertiles, and a multivariate logistic regression analysis was applied to assess the usefulness of the ABI and ba-PWV measurements as markers of cognitive impairment both crude and adjusted conditions for confounding factors (age, sex, amount of education, smoking status, habitual alcohol intake, body mass index, LDL-cholesterol, tryglyceride, HbA1c, systolic blood pressure, pulse pressure) [22]. The data were 
analyzed using the SPSS-PC-software for Windows, Version 12.0

\section{Results}

\section{Sample characteristics}

The subjects were divided into 2 groups according to their MMSE scores (poor cognition, MMSE $\% 23, \mathrm{n}=41$; control, $\mathrm{MMSE}^{\wedge} 24, \mathrm{n}=347$ ). The clinical characteristics of subjects are listed in Table 1. Subjects with poor cognition were significantly older and less educated than the control group. No other differences on all the other characteristics including the ba-PWV were observed.

\section{Effect of $A B I, P W V$ and education on cognitive decline}

The subjects were grouped according to their ABI tertile (first quartile 0.66 to $1.08, \mathrm{n}=129$; second tertile 1.08 to $1.15, \mathrm{n}=130$; third tertile 1.15 to $1.59, \mathrm{n}=129)$ and according to their ba-PWV tertile (first tertile 1159 to $1615 \mathrm{~cm} / \mathrm{s}, \mathrm{n}=129$; second tertile 1616 to $1895 \mathrm{~cm} / \mathrm{s}, \mathrm{n}=$ 130; third tertile 1899 to $3352 \mathrm{~cm} / \mathrm{s}, \mathrm{n}=129$ ). Table 2 presents the results of the multivariate logistic regression analysis used to assess the usefulness of the $\mathrm{ABI}$ and the brachial-ankle PWV as markers of the cognitive impairment. After adjusting for confounding factors, the $1^{\text {st }}$ quartile of $\mathrm{ABI}(\mathrm{OR}=3.19,95 \% \mathrm{CI}=1.30$ to 7.82$)$ was shown to be an independent risk factors. In crude odds ratio, the $1^{\text {st }}$ quartile of $\mathrm{ABI}(\mathrm{OR}=2.18,95 \% \mathrm{CI}=0.98$ to 4.87) approached statistical significance. Under both crude and adjusted conditions, amount of education consistently showed lower risk of cognitive impairment.
There were no other significant risk factors of cognitive decline.

\section{Discussion}

The ABI and PWV are the markers of atherosclerosis and arterial stiffness respectively. In the present research, their associations with cognitive impairment were examined through cross-sectional assessment among community-dwelling population. The cognitive decline was found to be 3.19 times more prevalent among subjects in the 1st tertile of ABI (0.66 to 1.08). On the other hand, no PWV tertiles showed an association with cognitive impairment in either crude or adjusted conditions. This research is considered as the report suggesting a usefulness of ABI and PWV as markers of cognitive function in a community-dwelling population.

The measurement of the $\mathrm{ABI}$ provides one of the most practical means to objectively assess the presence of atherosclerosis. A number of previous studies $[4,8,9]$ suggested that, even at subclinical levels, atherosclerosis is associated with an increased risk of progressive cognitive decline and $\mathrm{ABI}$ might be of clinical value in identifying older people who are at increased risk of cognitive impairment. The mechanism leading to the association between $\mathrm{ABI}$ and cognitive function still remains unclear. However, because ABI is a marker of structural and functional changes in the arterial vessels, several mechanisms may explain an association between atherosclerosis and dementia.

One explanation is that sub-clinical cerebrovascular disease, such as silent cerebral infarctions may mediate

\section{Table 1: Demographic Characteristics of the Subjects}

\begin{tabular}{|c|c|c|}
\hline & poor cognition $(n=41)$ & control $(n=347)$ \\
\hline MMSE* & $21.5 \pm 1.8$ & $28.0 \pm 2.0$ \\
\hline Age (years old) * & $70.1 \pm 4.9$ & $68.3 \pm 5.6$ \\
\hline Sex & male 21 , female 20 & male 118 , female 229 \\
\hline Amount of education (years) * & $9.3 \pm 1.9$ & $10.4 \pm 2.1$ \\
\hline Current smoking (0; no, 1 ; yes) & Yes 3 , No 38 & Yes 30, No 317 \\
\hline Habitual alcohol intake $(0 ;$ no, 1 ; yes) & Yes 22, No 19 & Yes 131, No 216 \\
\hline Body mass index $\left(\mathrm{kg} / \mathrm{m}^{2}\right)$ & $23.5 \pm 3.3$ & $23.3 \pm 2.9$ \\
\hline LDL-cholesterol (mmol/L) & $116.1 \pm 26.3$ & $118.9 \pm 24.4$ \\
\hline Triglyceride $(\mathrm{mmol} / \mathrm{L})$ & $92.4 \pm 47.4$ & $92.6 \pm 50.9$ \\
\hline $\mathrm{HbA1c}(\%)$ & $5.4 \pm 0.8$ & $5.3 \pm 0.6$ \\
\hline Systolic blood pressure $(\mathrm{mmHg})$ & $137.6 \pm 17.8$ & $136.8 \pm 17.3$ \\
\hline Pulse pressure (mmHg) & $57.0 \pm 12.1$ & $57.2 \pm 12.0$ \\
\hline Ankle-Brachial pressure index & $1.09 \pm 0.08$ & $1.11 \pm 0.09$ \\
\hline Pulse Wave Velocity (mm/s) & $1839 \pm 413$ & $1737 \pm 298$ \\
\hline
\end{tabular}

Values are mean \pm S.D. Student's unpaired $t$ test for continuous variables or chi-square test for categorical variables was used to evaluate the differences between poor cognition (MMSE\%23) and control (MMSE^24). * $p<0.05$ 
Table 2: Risk factors associated with having lower MMSE $(<24)$ estimated by logistic regression analysis.

\begin{tabular}{|c|c|c|c|c|c|}
\hline Parameter & & Crude odds ratio & p Value & Adjusted odds ratio & p Value \\
\hline Amount of education (years) & & $0.78(0.66-0.91)$ & $<0.01$ & $0.78(0.65-0.93)$ & $<0.01$ \\
\hline \multicolumn{6}{|l|}{ Ankle-Brachial pressure index } \\
\hline $1 \mathrm{st}$ & Tertile & $2.18(0.98-4.87)$ & $<0.10$ & $3.19(1.30-7.82)$ & $<0.05$ \\
\hline 2nd & Tertile & $1.10(0.45-2.69)$ & N.S. & $1.37(0.54-3.48)$ & N.S. \\
\hline $3 r d$ & Tertile & 1.00 & & 1.00 & \\
\hline \multicolumn{6}{|l|}{ Pulse Wave Velocity } \\
\hline $1 \mathrm{st}$ & Tertile & 1.00 & & 1.00 & \\
\hline 2nd & Tertile & $1.57(0.68-3.63)$ & N.S. & $1.43(0.48-4.23)$ & N.S. \\
\hline $3 r d$ & Tertile & $1.67(0.73-3.83)$ & N.S. & $1.50(0.58-3.85)$ & N.S. \\
\hline
\end{tabular}

the association between atherosclerosis and cognitive impairment. In the present research, brain imaging was not carried out on subjects, thus this was not evaluated. Also atherosclerosis may induce cerebral hypo-perfusion leading to cerebral hypoxia. These conditions may destabilize neurons and synapses and evolve in a neurodegenerative process characterized by formation of senile plaques, neurofibrillary tangles, and amyloid angiography $[5,23,24]$. Another explanation is that atherosclerosis and cognitive decline may share common genetic or environmental risk factors such as smoking, hypertension and hyper-lipidemia in the causal pathway [25]. The ABI measured in this study may reflect behaviour in early life that in turn influenced cognitive function. Although adjustment for cardiovascular risk factors in our study did not change the association between atherosclerosis and cognitive impairment as expected, this explanation still cannot be excluded.

In this study, we did not find an association between PWV and risk of cognitive impairment. Some findings obtained from other studies were found to support our results. For example, Dhoat et al [26] did not find a difference of PWV between dementia and control groups. In a large study of older Dutch population [27], arterial stiffness was not identified as an independent risk factor of cognitive decline neither. However, a number of studies [14-16] showed inverse relationships between PWV and MMSE. The brachial-ankle PWV (ba-PWV), which reflects left ventricular structure and function, was used in the present research to assess the arterial stiffness, whereas the carotid-femoral PWV (cf-PWV), a marker of arterial stiffness over the central elasticity was used in various studies. Ba-PWV is affected by not only central elastic arteries but also peripheral muscular ones [28]. Although ba-PWV correlates well with cf-PWV with a correlation coefficient around 0.75-0.87 [20,29] and cfPWV is the main determinant of ba-PWV [29], there is a substantial difference between the two techniques over the range of measurement [30].

In order to assess cognitive status, the MMSE was employed in this study. The MMSE score does not always reflect the cognitive function exactly, as it is sometimes known to be influenced by the education level of the subject. In the present study, the subjects with a normal MMSE score were significantly more educated than those with impaired MMSE scores, and the amount of education was found to have significant association with the MMSE score. This finding was consistent with that of previous reports. In Baltimore longitudinal Study of aging [17], persons with higher baseline PWV also exhibited prospective decline on tests of verbal learning and delayed recall, nonverbal memory, and the Blessed I-M-C Test. However, the same test did not show the association between PWV and MMSE.

As all subjects were volunteers who had interests in their health, they may be healthier than those who were not involved in this study. Thus, those who were not would be more likely to do poorly on a community-based cognitive assessment [31]. A "selective bias" must also be considered in studies of older persons. Those with severe arterial stiffness might not live to old age. However, the current findings indicate that a low ABI is an independent risk factor for cognitive impairment.

In the present study, the brain imaging was not carried out, thus Alzheimer's disease (AD) was not excluded completely. The aetiology of $\mathrm{AD}$ is different from vascular-type dementia. Including subjects with $\mathrm{AD}$ could obscure the association between vascular factors and cognitive function. Nevertheless, the result of this study, which showed cognitive impairment in subjects with lower ABI, suggests the predictive value of $\mathrm{ABI}$. 


\section{Conclusions}

In this study, we determined the association between ABI and PWV to cognitive impairment in cross-sectional study. A score in the lowest ABI tertile is an independent risk factor for cognitive impairment, whereas a score in a high ba-PWV tertile was not. However, we are not able to completely rule out an association between increased PWV and risk of cognitive impairment due to a number of factors including the measurement site of PWV, use of the MMSE, having a relatively small sample size and the possibility of a selection bias. Furthermore, a comparison between ABI and PWV was not performed in the longitudinal situation. There is still no cure for dementia, and the risk factors of cognitive decline must be identified to delay its progression. Therefore, further investigation is needed to obtain clear associations between them in the future.

\section{Competing interests}

The authors declare that they have no competing interests.

\section{Authors' contributions}

NS conceived the study, designed the study, conducted the statistical analysis, interpreted the data and wrote the initial draft of the manuscript. SK had full access to all of the data in the study and takes responsibility for the integrity of the data and the accuracy of the data analysis. SK, SN and NYF contributed to study design and assisted in drafting the manuscript. TU and IT completed initial survey construction, recruitment of participants. AK, YS, KD and MM participated in the data collection, and the interpretation of the results. All authors have approved the manuscript

\section{Acknowledgements}

The authors would like to thank Shoko Tsuchimine and all coworkers of the Iwaki Health Promotion Project for their skilful contributions to the data collection and management. This work was partly supported by a grant from Hirosaki Research Institute for the neurosciences.

\section{Author Details}

1Department of Psychiatry, Hirosaki-Aiseikai Hospital, Hirosaki, Japan, 2Department of Neuropsychiatry, Hirosaki University School of Medicine, Hirosaki, Japan and ${ }^{3}$ Department of Social Medicine, Hirosaki University School of Medicine, Hirosaki, Japan

Received: 17 January 2010 Accepted: 10 June 2010

Published: 10 June 2010

\section{References}

1. Gill TM, Richardson ED, Tinetti ME: Evaluating the risk of dependence in activities of daily living among community-living older adults with mild to moderate cognitive impairment. J Gerontol A Biol Sci Med Sci 1995, 50(5):235-241.

2. Weiler PG, Lubben JE, Chi I: Cognitive impairment and hospital use. Am J Public Health 1991, 81(9):1153-1157.

3. Liu IY, LaCroix AZ, White LR, Kittner SJ, Wolf PA: Cognitive impairment and mortality: a study of possible confounders. Am J Epidemio/ 1990, 132(1):136-143.

4. Price JF, McDowell S, Whiteman MC, Deary IJ, Stewart MC, Fowkes FG: Ankle brachial index as a predictor of cognitive impairment in the general population: ten-year follow-up of the Edinburgh Artery Study. J Am Geriatr Soc 2006, 54(5):763-769.

5. de la Torre JC: Is Alzheimer's disease a neurodegenerative or a vascular disorder? Data, dogma, and dialectics. Lancet Neurol 2004, 3(3):184-190.

6. Caruana MF, Bradbury AW, Adam DJ: The validity, reliability, reproducibility and extended utility of ankle to brachial pressure index in current vascular surgical practice. Eur J Vasc Endovasc Surg 2005, 29(5):443-451.
7. Drouet L: Atherothrombosis as a systemic disease. Cerebrovasc Dis 2002, 13(Suppl 1):1-6.

8. Haan MN, Shemanski L, Jagust WJ, Manolio TA, Kuller L: The role of APOE epsilon4 in modulating effects of other risk factors for cognitive decline in elderly persons. Jama 1999, 282(1):40-46.

9. Laurin D, Masaki KH, White LR, Launer LJ: Ankle-to-brachial index and dementia: the Honolulu-Asia Aging Study. Circulation 2007, 116(20):2269-2274.

10. Breteler MM, Claus JJ, Grobbee DE, Hofman A: Cardiovascular disease and distribution of cognitive function in elderly people: the Rotterdam Study. Bmi 1994, 308(6994):1604-1608.

11. Mizushima Y, Oobasawa H, Yoshida S, Irie H, Urata T, Shimoda H: Pulse wave velocity in persons with vascular dementia. J Am Geriatr Soc 2003, 51(9):1329-1330.

12. Nagai K, Akishita M, Machida A, Sonohara K, Ohni M, Toba K: Correlation between pulse wave velocity and cognitive function in nonvascular dementia. J Am Geriatr Soc 2004, 52(6):1037-1038.

13. Hanon $O$, Haulon S, Lenoir H, Seux ML, Rigaud AS, Safar M, Girerd X, Forette F: Relationship between arterial stiffness and cognitive function in elderly subjects with complaints of memory loss. Stroke 2005 , 36(10):2193-2197.

14. Fujiwara $Y$, Chaves $P H$, Takahashi $R$, Amano H, Yoshida H, Kumagai S, Fujita K, Wang DG, Shinkai S: Arterial pulse wave velocity as a marker of poor cognitive function in an elderly community-dwelling population. $J$ Gerontol A Biol Sci Med Sci 2005, 60(5):607-612.

15. Scuteri A, Brancati AM, Gianni W, Assisi A, Volpe M: Arterial stiffness is an independent risk factor for cognitive impairment in the elderly: a pilot study. J Hypertens 2005, 23(6):1211-1216.

16. Fukuhara M, Matsumura K, Ansai T, Takata Y, Sonoki K, Akifusa S, Wakisaka M, Hamasaki T, Fujisawa K, Yoshida A, Fujii K, lida M, Takehara T: Prediction of cognitive function by arterial stiffness in the very elderly. Circ J 2006, 70(6):756-761

17. Waldstein SR, Rice SC, Thayer JF, Najjar SS, Scuteri A, Zonderman AB: Pulse pressure and pulse wave velocity are related to cognitive decline in the Baltimore Longitudinal Study of Aging. Hypertension 2008, 51(1):99-104.

18. Scuteri A, Tesauro M, Appolloni S, Preziosi F, Brancati AM, Volpe M: Arterial stiffness as an independent predictor of longitudinal changes in cognitive function in the older individual. J Hypertens 2007, 25(5):1035-1040.

19. Koji Y, Tomiyama H, Ichihashi H, Nagae T, Tanaka N, Takazawa K, Ishimaru S, Yamashina A: Comparison of ankle-brachial pressure index and pulse wave velocity as markers of the presence of coronary artery disease in subjects with a high risk of atherosclerotic cardiovascular disease. Am J Cardio/ 2004, 94(7):868-872.

20. Yamashina A, Tomiyama H, Takeda K, Tsuda H, Arai T, Hirose K, Koji Y, Hori S, Yamamoto Y: Validity, reproducibility, and clinical significance of noninvasive brachial-ankle pulse wave velocity measurement. Hypertens Res 2002, 25(3):359-364.

21. Folstein MF, Folstein SE, McHugh PR: "Mini-mental state". A practical method for grading the cognitive state of patients for the clinician. J Psychiatr Res 1975, 12(3):189-198.

22. Mickey RM: The magnitude of the difference of crude and adjusted log odds ratios. Communications in Statistics - Theory and Methods 2005, 16(11):3403-3415.

23. Qiu C, Winblad B, Fratiglioni L: The age-dependent relation of blood pressure to cognitive function and dementia. Lancet Neurol 2005, 4(8):487-499.

24. van Oijen M, de Jong FJ, Witteman JC, Hofman A, Koudstaal PJ, Breteler MM: Atherosclerosis and risk for dementia. Ann Neurol 2007, 61(5):403-410.

25. Casserly I, Topol E: Convergence of atherosclerosis and Alzheimer's disease: inflammation, cholesterol, and misfolded proteins. Lancet 2004, 363(9415):1139-1146.

26. Dhoat S, Ali K, Bulpitt CJ, Rajkumar C: Vascular compliance is reduced in vascular dementia and not in Alzheimer's disease. Age Ageing 2008, 37(6):653-659.

27. Poels MM, van Oijen M, Mattace-Raso FU, Hofman A, Koudstaal PJ, Witteman JC, Breteler MM: Arterial stiffness, cognitive decline, and risk of dementia: the Rotterdam study. Stroke 2007, 38(3):888-892. 
28. Yu WC, Chuang SY, Lin YP, Chen CH: Brachial-ankle vs carotid-femoral pulse wave velocity as a determinant of cardiovascular structure and function. J Hum Hypertens 2008, 22(1):24-31.

29. Sugawara J, Hayashi K, Yokoi T, Cortez-Cooper MY, DeVan AE, Anton MA, Tanaka H: Brachial-ankle pulse wave velocity: an index of central arterial stiffness? J Hum Hypertens 2005, 19(5):401-406.

30. Choi JC, Lee JS, Kang SY, Kang JH, Bae JM, Lee DH: Limitation of brachialankle pulse wave velocity in assessing the risk of stroke: importance of instantaneous blood pressure. Cerebrovasc Dis 2009, 27(5):417-425.

31. Launer $L$, Wind AW, Deeg DJ: Nonresponse pattern and bias in a community-based cross-sectional study of cognitive functioning among the elderly. Am J Epidemiol 1994, 139(8):803-812.

\section{Pre-publication history}

The pre-publication history for this paper can be accessed here: http://www.biomedcentral.com/1471-244X/10/46/prepub

doi: $10.1186 / 1471-244 X-10-46$

Cite this article as: Sugawara et al., Comparison of ankle-brachial pressure index and pulse wave velocity as markers of cognitive function in a community-dwelling population BMC Psychiatry 2010, 10:46

Submit your next manuscript to BioMed Central and take full advantage of:

- Convenient online submission

- Thorough peer review

- No space constraints or color figure charges

- Immediate publication on acceptance

- Inclusion in PubMed, CAS, Scopus and Google Scholar

- Research which is freely available for redistribution

Submit your manuscript at www.biomedcentral.com/submit
Ciomed Central 